\title{
Neglected Achilles tendon rupture with central insertional plantaris tendon hypertrophy: two cases
}

\author{
Ronald Boer · Bart A. Swierstra • \\ Cees C. P. M. Verheyen
}

Received: 12 June 2008/Accepted: 17 February 2009/Published online: 10 March 2009

(C) Springer-Verlag 2009

\begin{abstract}
A neglected Achilles tendon rupture is often characterized by muscle weakness and an overlengthened repair by scar tissue. Reconstructive surgery is usually performed taking into account the patient's required level of function. Two surgical cases of neglected Achilles tendon rupture are presented in this article. In both instances it was expected that central fibrosis, possibly after neglected tendon rupture, would be found. However, after longitudinal opening of the tendons, a thickened plantaris tendon was evident at the insertion on the calcaneus in both cases. This hypertrophic tendon occupied most of the diameter of the Achilles tendon. Due to partial or complete rupture of the Achilles tendon, there was notable weakening and tendon transfer-augmentation was performed. A thickened plantaris tendon as a reaction to a neglected rupture of the Achilles tendon is a rare presentation. It can be detected preoperatively by MRI and subsequently preoperative planning can be optimized.
\end{abstract}

Keywords Achilles tendinopathy · Achilles tendinosis · Plantaris tendon hypertrophy .

Neglected Achilles tendon rupture

R. Boer - C. C. P. M. Verheyen

Isala Clinics, De Weezenlanden Hospital,

Groot Wezenland 20, Zwolle, The Netherlands

B. A. Swierstra

Sint Maartenskliniek, Hengstdal 3,

Nijmegen, The Netherlands

R. Boer ( $\square)$

Conkers, Pilgrims Lane, Newton, Rugby,

Warwickshire CV23 0ED, UK

e-mail: ronaldboer@btinternet.com

\section{Introduction}

The most commonly used definition of a neglected Achilles tendon rupture is an existing rupture of more than 4 weeks duration [2]. The history of neglected Achilles tendon rupture is often characterized by an overlengthened spontaneous repair by scar tissue. The accompanying weakness of the calf muscle can be treated by several reconstructive procedures. If, after excising the scar tissue, the gap is too big to bridge, a transfer-augmentation graft can be necessary. During two such reconstructions, we observed a hollowed, fibrosed Achilles tendon, which was filled centrally with a hypertrophic plantaris tendon. As far as we are aware, there are no other publications detailing the presentation of similar cases.

\section{Case reports}

\section{Case 1}

A 56-year-old, fit and healthy badminton player experienced pain around his left Achilles tendon after a bad fall. Following this injury, weight-bearing was not possible and the Achilles tendon became warm and swollen. A course of physiotherapy did not improve the situation and he was unable to continue playing badminton. This patient presented to our clinic 7 months after his injury complaining of difficulty in walking. On examination, we observed a thickened and painful left Achilles tendon and reduced plantar-flexor strength. A substantial rupture of the tendon, due to pre-existing tendinosis [3,4] of the tendon was considered. An MRI scan showed a thickened Achilles tendon with severe degenerative changes over $6-7 \mathrm{~cm}$, suggesting chronic tendinopathy [4]. At the time it was not 
noted by either radiologist or the surgeon that most of the central part of the Achilles tendon was filled with a thickened plantaris tendon (Fig. 1a, b).

The history suggested a substantial tear of the Achilles tendon and a surgical debridement with a flexor hallucis longus transfer-augmentation graft was planned. When the Achilles tendon was exposed, it appeared fibrosed, without a well-defined paratenon. After opening the tendon in a longitudinal manner over its degenerative part, a hollowed tendon became evident. The Achilles tendon was filled centrally with a hypertrophic plantaris tendon, instead of degenerative fibrous tissue. The plantaris tendon was still completely separated from the remaining Achilles tendon (Fig. 2a). Debridement and transfer-augmentation followed (Fig. 2b). The flexor hallucis longus tendon was divided just distal of Henry's knot with a tenodesis of the distal end to the flexor digitorum longus tendon to keep some active flexion in the hallux.

Biopsies taken from the Achilles tendon during surgery showed abnormal collagen with mucoid degeneration, hypercellularity and vascular infiltration, all compatible with peritendinitis and tendinosis. There was no evidence of an inflammatory reaction; few lymphocytes and no neutrophils were seen on microscopic examination. Deposits of hemosiderin were noted throughout the specimen, possibly caused by bleeding after rupture of the tendon.

\section{Case 2}

A 53-year-old man presented with a longstanding history of Achilles tendinopathy, which had been treated conservatively, including one steroid injection. One year prior to presentation, he had experienced acute pain around his left Achilles tendon, with impaired function, while playing tennis. He could no longer run and had difficulty walking up and down stairs. He also complained of morning stiffness and pain. On examination, there was atrophy of the calf muscle; toe walking was not possible and dorsiflexion
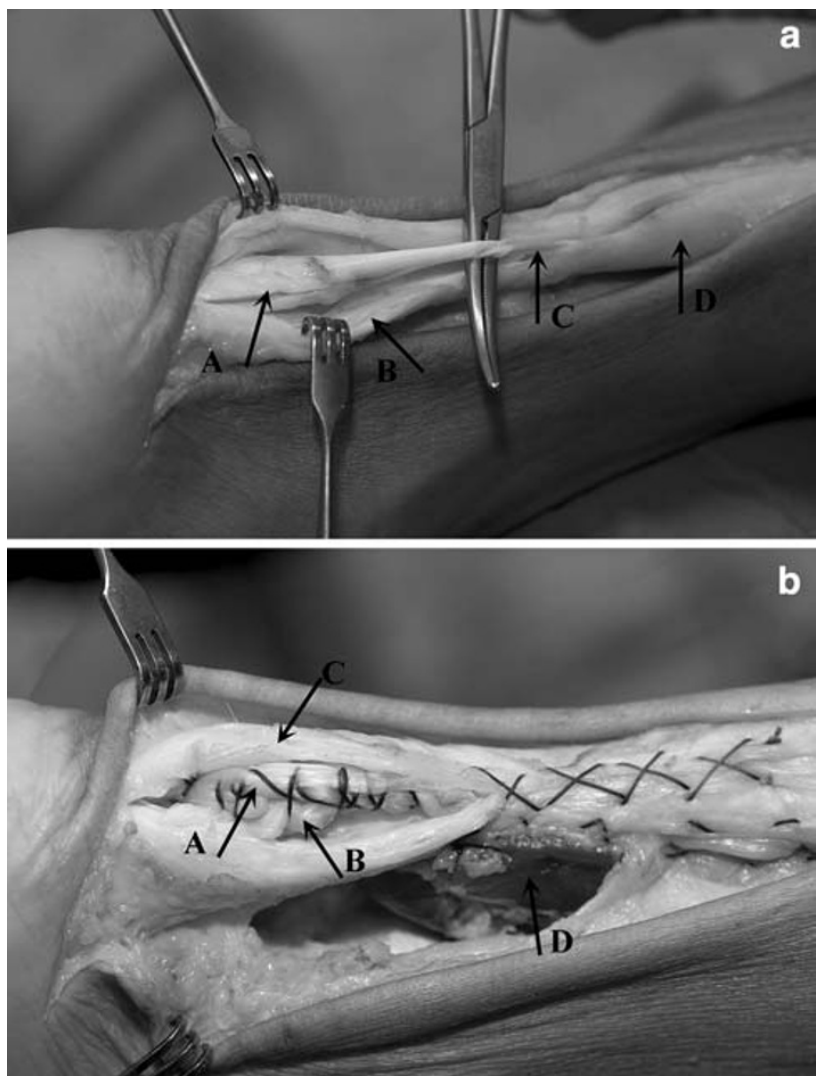

Fig. 2 a Perioperative findings in Case 1: A Centrally thickened plantaris tendon; $B$ Degenerative Achilles tendon; $C$ Normal plantaris tendon; $D$ Normal Achilles tendon. b Postoperative result after augmentation grafting of the Achilles tendon: $A$ Augmentation-graft with flexor hallucis longus tendon; $B$ Incorporated plantaris tendon; $C$ Achilles tendon; $D$ Muscle belly of flexor hallucis longus adjacent to the reconstruction to improve blood supply
Fig. 1 a Coronal section through the distal Achilles tendon. The MRI scan reveals the centrally thickened plantaris tendon $2 \mathrm{~cm}$. above the insertion at the calcaneus (within arrows). The maximum diameter in the frontal plane is $2.1 \mathrm{~cm}$. A fluid film around the plantaris tendon is visible, suggesting no cross-links between the two tendons. b Sagittal MRI scan of the Achilles tendon (Case 1). Hypertrophic plantaris tendon (A) surrounded by degenerative Achilles tendon $(B)$. Healthy Achilles tendon is in sharp contrast with the degenerative part $(C)$
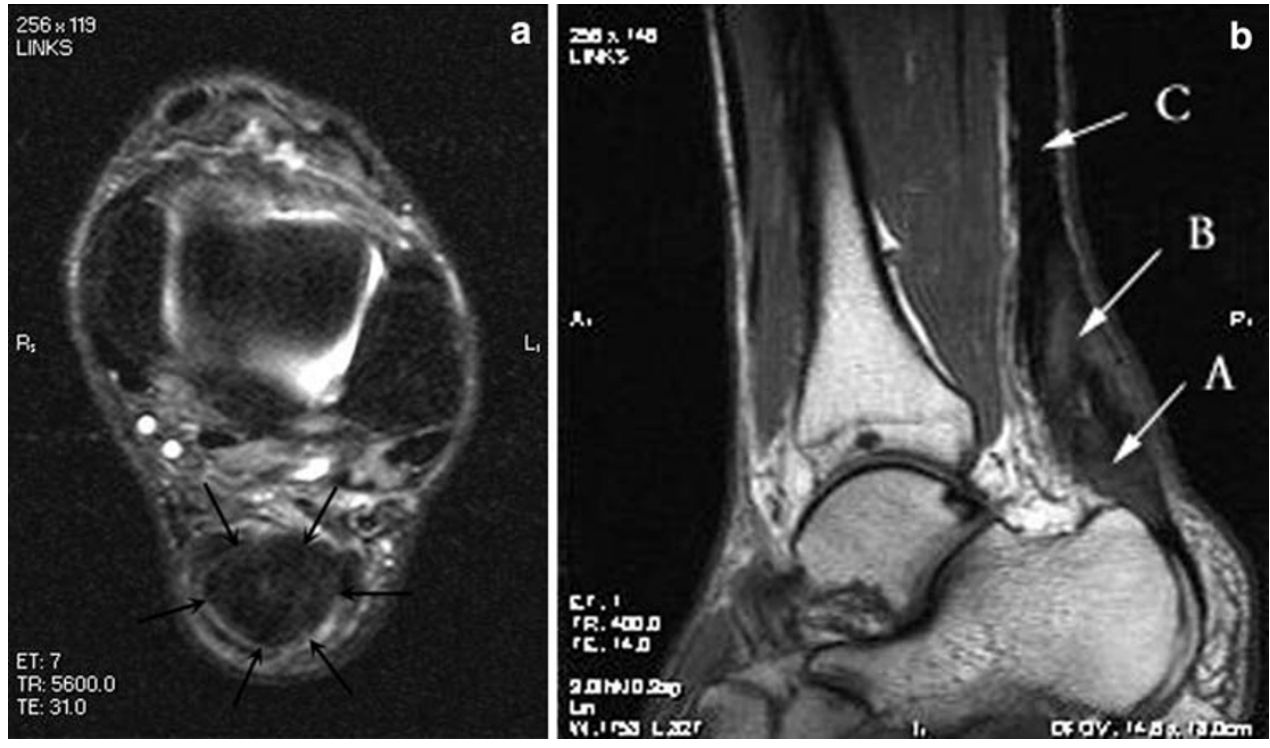


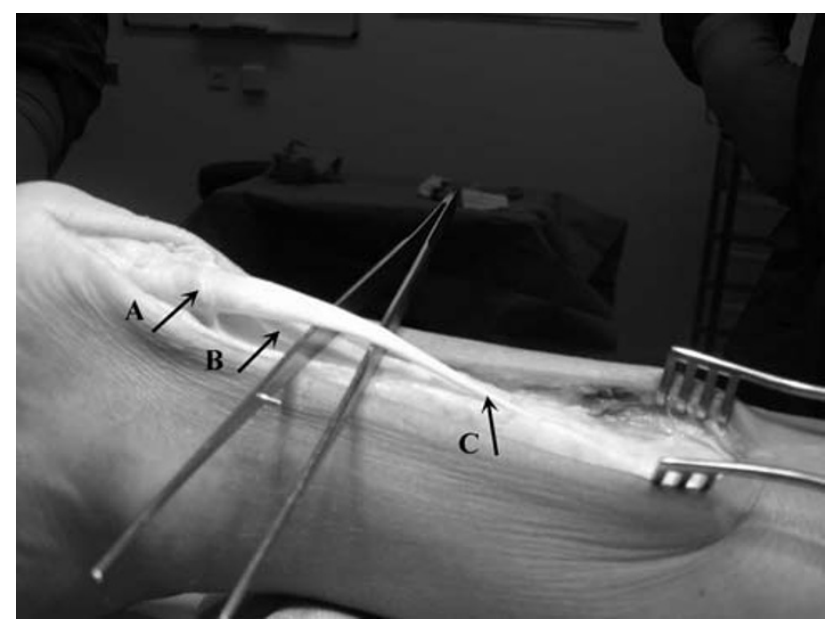

Fig. 3 Perioperative findings in Case 2: $A$ Thickened plantaris tendon; $B$ Degenerative Achilles tendon; $C$ Normal plantaris tendon

was increased on the left side. A neglected Achilles tendon rupture was suspected, and confirmed by ultrasound. Fibrosis bridging the tendon gap and tendinosis in the remaining tendon was also found. Hypertrophy of the plantaris tendon was not detailed in the radiology report. Operative reconstruction with augmentation graft was suggested and planned. After opening the Achilles tendon in a longitudinal manner, a hypertrophic plantaris tendon was clearly visible, which had no obvious cross-links with the remaining Achilles tendon (Fig. 3). Reconstruction by transfer-augmentation followed using the flexor digitorum tendon.

\section{Discussion}

Noninsertional Achilles tendinopathy is a disorder of the Achilles tendon, which can result in partial or complete rupture without any prior warning and is typically found in middle-aged athletes. In the case of a neglected rupture, patients will experience a functional deficit with loss of muscle power. In Achilles tendon rupture, the adjacent or integrated plantaris tendon is most often kept intact [5]. Only one report mentions a rupture of both tendons simultaneously [5]. In our cases, we hypothesize that the plantaris tendon substitutes for the ruptured Achilles tendon and causes scar tissue to form some kind of envelop around the plantaris tendon, resembling a tendon sheath. The increased load on the plantaris tendon leads to a compensatory hypertrophy, as described in experimental and clinical situations [1]. In both cases, there was clear evidence to support the hypothesis of a partial or complete tendon rupture. History and observed pathological changes in both presented cases indicate pre-existing tendinosis, with or without peritendinitis, although macroscopically no disorganized, fibrous, calcific tendinious tissue was found inside the tendon. The remains of the original Achilles tendon were minimal and the main bulk of the tendon consisted of a locally hypertrophic plantaris tendon. The plantaris tendon was still smooth and had no cross-links with the Achilles tendon proximal to the injury. Without a clear connection between the hypertrophic part of the plantaris tendon and the proximal Achilles tendon, load transfer between the two tendons is not possible. The partial, localized hypertrophy of the plantaris tendon at its insertion suggests the presents of such a load transfer, but in these cases no evidence of a macroscopic connection between the two tendons was found. It is also possible that the plantaris tendon takes over the function of the Achilles tendon completely, subsequently causing hypertrophy at the point of insertion. This localized hypertrophy will not strengthen an isolated plantaris tendon as a whole. A biomechanical explanation for localized tendon hypertrophy in these cases therefore remains unclear.

\section{Conclusion}

Two cases of neglected Achilles tendon rupture with an atypical presentation have been discussed. In both patients, the insertion of the Achilles tendon mainly consisted of a hypertrophic insertion of the plantaris tendon, surrounded by an inefficient, residual Achilles tendon. There is no known biomechanical explanation for this unusual presentation, but possibly it represents a natural compensatory repair mechanism initiated by a ruptured Achilles tendon.

Central plantaris tendon hypertrophy can be mistaken for advanced tendinosis of the Achilles tendon, but awareness of this phenomenon can directly lead to the correct diagnosis of neglected Achilles tendon rupture.

\section{References}

1. Buchanan CI, Marsh RL (2002) Effect of exercise on the biomechanical, biochemical and structural properties of tendons. Comp Biochem Physiol A Mol Integr Physiol 133(4):1101-1107

2. Gabel S, Manoli A 2nd (1994) Neglected rupture of the Achilles tendon. Foot Ankle Int 15(9):512-517

3. Maffulli N, Khan KM, Puddu G (1998) Overuse tendon conditions: time to change a confusing terminology. Arthroscopy 14(8):840 843

4. Puddu G, Ippolito E, Postacchini F (1996) A classification of Achilles tendon disease. Am J Sports Med 4(4):145-150

5. Vanderhooft E (1997) Concurrent rupture of the Achilles and plantaris tendons. Am J Orthop 26(12):857-858 